\title{
Designing Rules of Laser-Clad High-Entropy Alloy Coatings with Simple Solid Solution Phases
}

\author{
Yongfei Juan ${ }^{1} \cdot$ Jiao Zhang ${ }^{1} \cdot$ Yongbing Dai ${ }^{1} \cdot$ Qing Dong ${ }^{1} \cdot$ Yanfeng Han ${ }^{1}$
}

Received: 29 October 2019 / Revised: 10 December 2019 / Published online: 22 April 2020

(c) The Chinese Society for Metals (CSM) and Springer-Verlag GmbH Germany, part of Springer Nature 2020

\begin{abstract}
The criteria of process parameters $(\mu \leq 4)$, atomic size difference $(\varepsilon \leq 5.8, \delta \leq 11$ and $\alpha \leq 2)$, thermodynamic $\left(-14.5 \leq \Delta H_{\text {mix }} \leq 6.5\right.$ and $\left.\Omega \geq 1.8\right)$ in the prediction of the phase stability for laser-clad high-entropy alloy coatings are studied in detail. Besides, the criteria of valence electron concentration (VEC) applied to distinguish the stability of different solid solution phases are as follows: VEC $<7.65$ for simple BCC, VEC $\geq 7.65$ for simple face-centered cubic (FCC), $7.14<$ VEC $<7.78$ for dual-phase BCC and FCC. Among them, $\mu$ and $\varepsilon$ proposed firstly separate the phase stability of laserclad high-entropy alloy coatings quite precisely. The other modified criteria $\left(\delta, \alpha, \Delta H_{\text {mix }}, \Omega\right.$, VEC) are proved to be different from those of the high-entropy alloys synthesized by the traditional casting and smelting processes.
\end{abstract}

Keywords High-entropy alloys $\cdot$ Laser cladding $\cdot$ Phase stability $\cdot$ Criteria

\section{Introduction}

High-entropy alloys (HEAs), as a new alloy design concept, were proposed by Ye et al. [1] in 2004 and have attracted much attention owing to their unique phase formation ability [2-6]. According to the definition [7, 8], HEAs typically consist of at least five major elements with the atomic percentage between 5 and $35 \%$. It has been widely proved that the multi-component alloys were composed of solid solution phases such as face-centered cubic (FCC) and bodycentered cubic (BCC) rather than intermetallic compounds [9-15]. Huang et al. [16] reported that the phase transformation from FCC to hexagonal-close-packed (HCP) structure was observed in $\mathrm{CoCrFeMnNi}$ alloy when applied hydrostatic pressures up to $20 \mathrm{GPa}$, and the lattice asymmetric crossover before and after phase transition had been found under hydrostatic compression surroundings. In the work of Xiang et al. [17], MoNbFeCrV, MoNbFeCrTi, and MoNbFeVTi HEAs containing low thermal neutron absorption

Available online at http://link.springer.com/journal/40195.

Jiao Zhang

18817307579@163.com

1 Shanghai Key Lab of Advanced High-Temperature Materials and Precision Forming, Shanghai Jiao Tong University, Shanghai 200240, China cross-section elements were prepared by vacuum arc melting, in which a dominant body-centered cubic (BCC) phase was present in all these three HEAs. Ishizu et al. [18] discussed the comparison of cocktail effects of $T_{\mathrm{c}}$ among the BCC HEA superconductors, in which $\mathrm{Hf}_{21} \mathrm{Nb}_{25} \mathrm{Ti}_{15} \mathrm{~V}_{15} \mathrm{Zr}_{24}$ BCC HEA had been proved to be a new superconductor with the superconducting critical temperature $\left(T_{\mathrm{c}}\right)$ of $5.3 \mathrm{~K}$. Obviously, the existence of solid solution phase always plays a decisive role in the properties of HEAs. Therefore, accurately predicting the phase stability is extremely significant for the design of high-performance HEAs [19-21].

Hume-Rothery rules [22] have been widely applied in the phase studies of binary alloys. Based on the theory, Zhang et al. [23] proved that solid solution phases were easily formed when the difference of the atomic size $(\delta)$ is relatively small, the values of the mixed entropy $\left(\Delta S_{\text {mix }}\right)$ are relatively high, and the enthalpy of mixing $\left(\Delta H_{\text {mix }}\right)$ is either slightly positive or not very negative. After that, the significant results were widely confirmed and stretched in the other studies [24-28], in which the atomic packing parameter $\gamma$ applied to express the influences of the atomic size difference was proposed by Wang et al. [24], and then a new phenomenological model was raised to analyze the phase formation ability through the connection of lattice distortion and atomic size difference [25]. Besides, an optimized parameter $\Phi$ combining the effects of the entropy of mixing $\left(\Delta S_{\text {mix }}\right)$ and enthalpy of mixing $\left(\Delta H_{\text {mix }},\right)$ was put forward 
by Yang et al. [26]; the parameter VEC (valence electron concentration) which plays a decisive role in determining the phase formation of FCC and BCC for HEAs was discussed by Guo et al. [27, 28]. All these conclusions and the other unmentioned works [29-31] about the study of phase formation contribute significantly to the development of HEAs.

However, all of these researches have been mostly focused on the as-cast bulk material, and the phase formation studies about HEA coatings are relatively few. A lot of significant results have been discovered in the previous works, but there are still some defects such as micropore which caused by thermal expansion and contraction for the as-cast bulk HEAs cannot be completely avoided. What's more, precious metal elements (such as $\mathrm{Cr}, \mathrm{Co}, \mathrm{Ni}, \mathrm{Mo}$ ) are always essential to prepare HEAs, which must cause high cost and resources waste when applied traditional casting and smelting processes as the fabricated method [32-34].

Laser cladding has attracted much attention in recent years. Compared with the other traditional surface modification technologies such as spraying [35], electro-spark deposition [36], hot pressing sintering [37], laser cladding processes possess many remarkable advantages such as a good metallurgical bonding easily formed between the coatings and substrates with the thickness of the laser-clad coatings that can reach up to millimeter level simultaneously. AlCoCrFeNixNbC HEA coatings ( $x=10,20,30 \mathrm{wt} \%$ ) composed of simple BCC and FCC structure phases were prepared on the surface of Q235 steel by laser cladding [38], in which the addition of $\mathrm{NbC}$ particles caused a decrease in the fraction of FCC phase. The micro-hardness (525 HV) and the wear resistance of the coatings both improved significantly with the addition of NbC. Liu et al. [39] synthesized the AlCoCrFeNiTix ( $x=0,0.2,0.4,0.6,0.8,1.0)$ HEA coatings on AISI1045 steel by laser cladding. The analysis showed that the coatings were mainly composed of simple BCC phase, and the spinodal decomposition structures which enriched $\mathrm{Fe}-\mathrm{Cr}$ and $\mathrm{Al}-\mathrm{Ni}-\mathrm{Ti}$ were found in grains. Meanwhile, the passivation behavior of the coatings has been caused by the introduction of $\mathrm{Ti}$, in which the HEA coating with $x=1.0$ showed the best corrosion resistance.

What's more, the process parameters also take significant impacts on the phase stability of laser-clad coatings due to the characteristics of rapid heating and cooling for laser cladding process [40]. Khamidullin et al. [41] presented a mathematical modeling of substrate melting and the formation of the cladding layer during the laser cladding process. A phase-field method was applied to simulate the phase transition, and the influences of process parameters on the geometry of a laser-clad area and heat-affected zone were revealed. Riquelme et al. [42] demonstrated the effects of process parameters on the alloy properties, in which ZE41 magnesium alloy was used as the substrate and the cladding material was $\mathrm{Al}-35 \mathrm{wt} \% \mathrm{SiC}$. And it has been found that the effects of laser cladding parameters (laser power of $500-700 \mathrm{~W}$, scan speed of 3-17 mm/s, laser beam focal position) dramatically impact the clad geometry and dilution.

Unfortunately, most of the current studies about HEAs focus on traditional bulk materials and relatively few studies center on HEA coatings. Besides, the effects of laser cladding parameters on the phase stability of laser-clad HEA coatings have not been investigated. Therefore, it is a meaningful topic to explore the new phase stability criteria of laser-clad HEA coatings for the studies both of HEAs and laser cladding.

\section{Criteria Analysis}

\subsection{Factors of Process Parameters}

During the laser cladding process, high-energy-density laser beam is applied to melt the deposited materials and the substrate surface parts, and then the melted substrate parts diffuse into the coatings with the effects of fluid convective [43]. It is sure that a stronger melting of the substrates is brought about when the energy density is relatively high, which is conducive to the intensification of the metallurgical bonding between the substrate and coatings [44], and a great amount of energy accumulated in the molten pool, resulting in the composition of the substrate exceed the expected in the coatings $[45,46]$. On the contrary, the characteristic of rapid cooling rates for the laser cladding process is maximized when the laser energy density is relatively low. Nevertheless, much too fast cooling rates are not only conducive to the formation of solid solution phases which are expected for HEAs, but also contribute to the appearance of the glass phase due to the diffuse of the atoms is weakened seriously [47]. Meanwhile, a relatively weak bonding between substrates and coatings can be generated when the laser energy is deficient to melt the substrate surface parts and no enough time for the formation of high-quality metallurgical bonding.

Therefore, controlling laser energy density in a suitable range is extremely essential to prepare the superior performance laser-clad HEA coatings composed of simple solid solution phases. Based on the above analysis, parameter $\mu$ (the laser energy density) is proposed to assess the comprehensive effects of the laser parameters including laser power, scanning speed and spot size on the phase stability of laser-clad HEA coatings, and the calculation formula of $\mu$ can be given as follows:

$\mu=100 \times \frac{P}{S \cdot v}$.

where $\mu$ is the specific laser energy density, $P$ and $v$ are the laser power and the scanning speed, respectively. 
And it must be noted that the shapes of the laser-spot are not the same for different lasers, which means that it is not accurate enough to regard diameter or side length of the spot as the laser beam shape. Considering this point, the actual area $S$ is cited to measure the laser-energy concentrated area. Then the values of $\mu$ corresponding to different phase compositions are calculated and listed in Table 1.

\subsection{Factors of Dynamics}

\subsubsection{Atomic Size Difference of All Elements}

Through the analysis of the phase compositions and the corresponding alloy elements (Table 1), an interesting phenomenon can be found that the coatings composed of intermetallic (IM) compounds mostly contained B element. Is it just a coincidence? Hume-Rothery rules known as the remarkable achievement in solid solubility researches are applied to answer this question [22]. In these rules, the first one also the important one is the size effects of the component atoms that the formation ability of solid solution phase plays a major role when the atomic size difference is less than $15 \%$ [69, 70]. Then considering the atomic size of $\mathrm{B}(0.82 \AA)$ is small than those of the elements most frequently used in HEAs preparation such as $\mathrm{Fe}(1.25 \AA), \mathrm{Co}(1.248 \AA), \mathrm{Cr}(1.249 \AA)$ and $\mathrm{Ni}(1.246 \AA)$, the question raised is readily understood. However, Hume-Rothery rules have been widely applied in binary alloy systems, and they are still at a primary stage in the study of solid-solution solubility for HEAs [71-73].

Based on the definition of HEAs, the opportunities for the atoms to occupy the lattice sites should be close [1-8], which is not easy to be realized when the atomic size difference is too large. Moreover, serious lattice distortion and drastic increase in the alloy system energy is brought about along with a large atomic size difference. Therefore, a relatively low atomic size difference is helpful for the formation of high-performance HEAs with simple solid-solution phases. To characterize the atomic size difference for HEAs more clearly, population standard deviation (STDEVP) also known as the theoretical standard deviation [74] was cited. In the light of the mathematical definitions, relatively smaller values of STDEVP signify the measured data are consistent $[75,76]$, and larger ones indicate the data characteristics are highly dispersive. This numerical method which judges the dispersion degree among individuals is applied to compare the atomic size difference for HEAs in this work. Besides, the fact remains that the contents of all the elements are difficult to be same especially for the laser cladding process, which means that the influence of atomic size is mixed with that of the element contents on phase stability.

According to the above-mentioned analytical results, new parameter $\varepsilon$ is proposed to identify the atomic size difference for a given HEA system. And the calculation formula of $\varepsilon$ is given by:

$\varepsilon=100 \times \sqrt{\frac{1}{n} \times \sum_{i=1}^{n} c_{i}\left(r_{i}-\bar{r}\right)^{2}}$.

where $n$ signifies the number of the elements, $r_{i}$ and $c_{i}$ are the atomic radius and the element percent of the $i$ th element, respectively, and the average atomic radius $(\bar{r})$ in the alloy systems is given as:

$\bar{r}=\sum_{i=1}^{n} r_{i} c_{i}(3)$.

The calculated results of $\varepsilon$ corresponding to the laser-clad HEA coatings with different phase compositions are listed in Table 1. Meanwhile, the similar parameter atomic size difference $\delta$ has been widely used to predict the phase stability of HEAs in the other studies [14, 23, 24, 26, 28], and the calculation formula of $\delta$ is given by:

$\delta=100 \sqrt{\sum_{i=1}^{n} c_{i}\left(1-\frac{r_{i}}{\bar{r}}\right)^{2}}$.

where the meanings of $r_{i}, c_{i}$ and $\bar{r}$ are the same as those appeared in Eq. (2).

\subsubsection{Atomic Size Difference of Specific Elements}

The atomic size of all the elements is considered during the analytic processes both of $\varepsilon$ and $\delta$ when characterizing the solid-solution stability. However, it has been proved that solid-solution instability is fundamentally determined by the largest and the smallest atoms in the HEAs. Wang et al. [24] discussed the effects of atomic size difference on the topological instability of atomic packing in HEAs. The atomic packing effects in HEAs were quantitatively expressed by choosing the solid angles of atomic packing for the components with the largest atoms and the smallest atoms. The solid angles around the special atoms concerning the surrounding atoms are calculated by the following geometric formulas:

$\omega_{S}=1-\sqrt{\frac{\left(r_{S}+\bar{r}\right)^{2}-\bar{r}^{2}}{\left(r_{S}+\bar{r}\right)^{2}}}$.

$\omega_{L}=1-\sqrt{\frac{\left(r_{L}+\bar{r}\right)^{2}-r^{2}}{\left(\bar{r}_{S}+\bar{r}\right)^{2}}}$.

where $r_{\mathrm{S}}$ and $r_{\mathrm{L}}$ are the atomic radii of the smallest and largest atoms, respectively. 
Table 1 Calculated values of the nine criterions $\mu, \varepsilon, \delta, \gamma, \alpha, \Delta S_{\text {mix }}$, $\Delta H_{\text {mix }}, \Omega$ and VEC based on a large number of data obtained from the publicly reported references related to laser cladding, in which, intermetallic component phase was represented by IM, industrial pure iron is abbreviated to pure iron, magnesium is abbreviated to $\mathrm{Mg}$

\begin{tabular}{|c|c|c|c|c|c|c|c|c|c|c|c|c|}
\hline Substrates & Coatings or areas & $\mu$ & $\varepsilon$ & $\delta$ & $\gamma$ & $\alpha$ & $\Delta S_{\text {mix }}$ & $\Delta H_{\text {mix }}$ & $\Omega$ & VEC & Phase & References \\
\hline Q235 steel & $\mathrm{FeCoCrNi}$ & $3.5 \mathrm{~s}$ & - & 0.97 & - & - & 11.52 & -3.75 & 5.96 & 8.52 & fcc & [48] \\
\hline Pure iron & $\mathrm{AlCoCrFeNiTi}_{0.5}$ & - & - & 12.62 & 1.0086 & 1.50 & 14.43 & -14.07 & 1.81 & 7.43 & fcc & [49] \\
\hline A steel plate & CoCrFeMnNi & - & - & 0.48 & 1.0087 & 0.12 & 13.37 & -4.12 & 5.80 & 7.96 & fcc & {$[50]$} \\
\hline Q235 steel & $\mathrm{CoCrFeMnNi}$ & - & - & 0.32 & 1.0078 & 0.05 & 12.56 & -3.23 & 6.85 & 7.55 & fcc & {$[51]$} \\
\hline $\mathrm{CoCrFeNi}$ & $\mathrm{Al}_{0.15} \mathrm{CoCrFeNi}$ & - & 0.99 & 1.77 & 1.0069 & 0.27 & 12.42 & -5.48 & 4.14 & 8.04 & fcc & {$[52]$} \\
\hline $\mathrm{CoCrFeNi}$ & $\mathrm{Al}_{0.20} \mathrm{CoCrFeNi}$ & - & 1.11 & 1.97 & 1.0069 & 0.33 & 12.56 & -5.82 & 3.92 & 7.98 & fcc & {$[52]$} \\
\hline $\mathrm{CoCrFeNi}$ & $\mathrm{Al}_{0.24} \mathrm{CoCrFeNi}$ & - & 1.21 & 2.15 & 1.0326 & 0.39 & 12.68 & -6.25 & 3.67 & 7.91 & fcc & {$[52]$} \\
\hline $\mathrm{CoCrFeNi}$ & $\mathrm{Al}_{0.28} \mathrm{CoCrFeNi}$ & - & 1.29 & 2.30 & 1.0069 & 0.45 & 12.78 & -6.61 & 3.48 & 7.88 & $\mathrm{fcc}$ & {$[52]$} \\
\hline $\mathrm{CoCrFeNi}$ & $\mathrm{Al}_{0.28} \mathrm{CoCrFeNi}$ & - & 1.29 & 2.30 & 1.0069 & 0.45 & 12.78 & -6.63 & 3.48 & 7.85 & fcc & {$[52]$} \\
\hline $\mathrm{CoCrFeNi}$ & $\mathrm{Al}_{0.34} \mathrm{CoCrFeNi}$ & - & 1.41 & 2.50 & 1.0069 & 0.51 & 12.91 & -7.17 & 3.22 & 7.83 & $\mathrm{fcc}$ & {$[52]$} \\
\hline $\mathrm{CoCrFeNi}$ & $\mathrm{Al}_{0.37} \mathrm{CoCrFeNi}$ & - & 1.45 & 2.58 & 1.0069 & 0.54 & 12.96 & -7.31 & 3.16 & 7.79 & fcc & {$[52]$} \\
\hline 45\# steel & $\mathrm{FeCoCr}_{0.5} \mathrm{NiB}$ & 2.36 & 5.47 & 10.10 & 1.0062 & 2.14 & 12.23 & -10.28 & 2.21 & 8.22 & fcc & {$[53]$} \\
\hline $45 \#$ steel & $\mathrm{FeCoCrNiB}$ & 2.36 & 0.14 & 0.24 & 1.0061 & 0.05 & 11.05 & -2.77 & 7.20 & 8.67 & fcc & {$[53]$} \\
\hline $45 \#$ steel & $\mathrm{FeCoCr}_{1.5} \mathrm{NiB}$ & 2.36 & 0.15 & 0.27 & 1.0069 & 0.05 & 11.24 & -3.04 & 6.74 & 8.55 & fcc & {$[53]$} \\
\hline 45\# steel & $\mathrm{FeCoCr}_{2.0} \mathrm{NiB}$ & 2.36 & 0.14 & 0.25 & 1.0069 & 0.05 & 11.46 & -3.47 & 6.08 & 8.40 & fcc & {$[53]$} \\
\hline 45\# steel & $\mathrm{FeCoCr}_{3.0} \mathrm{NiB}$ & 2.36 & 0.15 & 0.26 & 1.0069 & 0.05 & 11.37 & -3.89 & 5.51 & 7.99 & fcc & {$[53]$} \\
\hline 45\# steel & $\mathrm{FeCrNiCoMnB}_{0.25}$ & 2.36 & 0.22 & 0.39 & 1.0087 & 0.07 & 13.20 & -3.05 & 8.19 & 8.11 & fcc & {$[54]$} \\
\hline 45\# steel & $\mathrm{FeCrNiCoMnB}{ }_{0.50}$ & 2.36 & 0.22 & 0.40 & 1.0087 & 0.07 & 13.03 & -2.66 & 9.21 & 8.34 & fcc & {$[54]$} \\
\hline 45\# steel & $\mathrm{FeCrNiCoMnB}_{0.75}$ & 2.36 & 0.22 & 0.40 & 1.0087 & 0.06 & 12.53 & -2.29 & 10.17 & 8.51 & fcc & {$[54]$} \\
\hline $45 \#$ steel & $\mathrm{FeCrCoNi}(\mathrm{SiC}-0)$ & 6.79 & 0.14 & 0.24 & 0.9085 & 0.05 & 11.05 & -2.77 & 7.20 & 8.67 & fcc & {$[55]$} \\
\hline 45\# steel & $\mathrm{FeCrCoNi}(\mathrm{SiC}-0-900)$ & 6.79 & 0.14 & 0.26 & 0.9085 & 0.05 & 10.77 & -2.59 & 7.49 & 8.81 & fcc & {$[55]$} \\
\hline $\mathrm{AZ} 31 \mathrm{Mg}$ & $\mathrm{AlCoCrCuFeNiSi}_{0.5}(\mathrm{ID})$ & 2.39 & 3.59 & 6.22 & 1.1691 & 1.48 & 13.94 & -11.86 & 1.78 & 7.65 & fcc & {$[56]$} \\
\hline $\mathrm{AZ} 31 \mathrm{Mg}$ & $\mathrm{AlCoCrCuFeNiSi}_{0.5}($ Shell $)$ & 2.39 & 3.71 & 6.41 & 1.1691 & 1.49 & 13.97 & -14.16 & 1.52 & 7.35 & fcc & {$[56]$} \\
\hline Q235 steel & $\mathrm{FeCoCrNiB}_{0.50}(\mathrm{~A})$ & 3.54 & 5.40 & 10.00 & 1.007 & 2.11 & 12.54 & -10.61 & 2.23 & 7.89 & fcc & {$[57]$} \\
\hline Q235 steel & $\mathrm{FeCoCrNiB}_{0.75}(\mathrm{~A})$ & 3.54 & 5.72 & 10.90 & 1.0071 & 2.74 & 12.62 & -10.70 & 2.17 & 7.71 & fcc & {$[57]$} \\
\hline Q235 steel & $\mathrm{FeCoCrNiB}(\mathrm{A})$ & 3.54 & 4.41 & 8.09 & 1.0061 & 1.46 & 11.91 & -7.67 & 2.87 & 8.19 & fcc & {$[57]$} \\
\hline Q235 steel & $\mathrm{FeCoCrNiB}_{1.25}(\mathrm{~A})$ & 3.54 & 5.00 & 9.21 & 1.0061 & 1.84 & 12.25 & -9.15 & 2.48 & 8.19 & fcc & {$[57]$} \\
\hline 904L steel & $\mathrm{CoCr}_{2} \mathrm{FeNi}(\mathrm{DR})$ & 0.91 & 0.54 & 0.97 & 1.007 & 0.34 & 11.14 & -3.99 & 5.21 & 7.96 & fcc & {$[58]$} \\
\hline 904L steel & $\mathrm{CoCr}_{2} \mathrm{FeNi}(\mathrm{ID})$ & 0.91 & 0.14 & 1.92 & 1.0069 & 0.05 & 11.03 & -4.00 & 5.13 & 7.76 & fcc & {$[58]$} \\
\hline 904L steel & $\mathrm{CoCr}_{2} \mathrm{FeTi}_{0.5} \mathrm{Ni}(\mathrm{DR})$ & 0.91 & 1.60 & 3.11 & 1.0069 & 0.39 & 11.71 & -5.82 & 3.81 & 7.63 & fcc & {$[58]$} \\
\hline 904L steel & $\mathrm{CoCr}_{2} \mathrm{FeTi}_{0.5} \mathrm{Ni}(\mathrm{ID})$ & 0.91 & 4.32 & 7.46 & 1.0068 & 1.55 & 12.25 & -14.53 & 1.61 & 7.30 & fcc & {$[58]$} \\
\hline 904L steel & $\mathrm{CoCr}_{2} \mathrm{FeTiNi}(\mathrm{DR})$ & 0.91 & 2.02 & 3.97 & 1.0069 & 0.59 & 11.71 & -6.58 & 3.36 & 7.55 & fcc & {$[58]$} \\
\hline 904L steel & $\mathrm{CoCr}_{2} \mathrm{FeTiNi(ID)}$ & 0.91 & 3.28 & 8.51 & 1.1908 & 1.23 & 12.65 & -12.82 & 1.73 & 7.78 & fcc & {$[58]$} \\
\hline CoCrFeNi & $\mathrm{Al}_{0.41} \mathrm{CoCrFeNi}$ & - & 1.52 & 2.70 & 1.0069 & 0.58 & 13.03 & -7.72 & 2.99 & 7.78 & $\mathrm{fcc}+\mathrm{bcc}$ & {$[52]$} \\
\hline $\mathrm{CoCrFeNi}$ & $\mathrm{Al}_{0.48} \mathrm{CoCrFeNi}$ & - & 1.62 & 2.87 & 1.1069 & 0.65 & 13.13 & -8.24 & 2.80 & 7.68 & $\mathrm{fcc}+\mathrm{bcc}$ & {$[52]$} \\
\hline $\mathrm{CoCrFeNi}$ & $\mathrm{Al}_{0.52} \mathrm{CoCrFeNi}$ & - & 1.66 & 2.95 & 1.1069 & 0.67 & 13.17 & -8.45 & 2.73 & 7.64 & $f c c+b c c$ & {$[52]$} \\
\hline $\mathrm{CoCrFeNi}$ & $\mathrm{Al}_{0.54} \mathrm{CoCrFeNi}$ & - & 1.99 & 3.60 & 1.1077 & 0.79 & 13.10 & -8.37 & 2.68 & 7.51 & $f c c+b c c$ & {$[52]$} \\
\hline $\mathrm{CoCrFeNi}$ & $\mathrm{Al}_{0.69} \mathrm{CoCrFeNi}$ & - & 1.85 & 3.268 & 1.1067 & 0.78 & 13.30 & -9.52 & 2.41 & 7.48 & $f c c+b c c$ & {$[52]$} \\
\hline $\mathrm{CoCrFeNi}$ & $\mathrm{Al}_{0.69} \mathrm{CoCrFeNi}$ & - & 1.85 & 3.268 & 1.1067 & 0.78 & 13.30 & -9.52 & 2.41 & 7.48 & $f c c+b c c$ & {$[52]$} \\
\hline $\mathrm{CoCrFeNi}$ & $\mathrm{Al}_{0.71} \mathrm{CoCrFeNi}$ & - & 1.86 & 3.29 & 1.1067 & 0.79 & 13.31 & -9.60 & 2.39 & 7.47 & $f c c+b c c$ & {$[52]$} \\
\hline $\mathrm{CoCrFeNi}$ & $\mathrm{Al}_{0.78} \mathrm{CoCrFeNi}$ & - & 1.92 & 3.40 & 1.1067 & 0.82 & 13.34 & -9.88 & 2.31 & 7.37 & $f c c+b c c$ & {$[52]$} \\
\hline $\mathrm{CoCrFeNi}$ & $\mathrm{Al}_{0.82} \mathrm{CoCrFeNi}$ & - & 1.96 & 3.46 & 1.1066 & 0.83 & 13.36 & -10.26 & 2.22 & 7.36 & $f c c+b c c$ & {$[52]$} \\
\hline $\mathrm{CoCrFeNi}$ & $\left.\mathrm{Al}_{0.83} \mathrm{CoCrFe}\right] \mathrm{Ni}$ & - & 1.96 & 3.46 & 1.1066 & 0.83 & 13.36 & -10.24 & 2.22 & 7.35 & $f c c+b c c$ & {$[52]$} \\
\hline $\mathrm{CoCrFeNi}$ & $\left.\mathrm{Al}_{0.85} \mathrm{CoCrFe}\right] \mathrm{Ni}$ & - & 1.97 & 3.48 & 1.1066 & 0.84 & 13.36 & -10.31 & 2.20 & 7.33 & $f c c+b c c$ & {$[52]$} \\
\hline $\mathrm{CoCrFeNi}$ & $\left.\mathrm{Al}_{0.86} \mathrm{CoCrFe}\right] \mathrm{Ni}$ & - & 1.98 & 3.50 & 1.1066 & 0.84 & 13.36 & -10.38 & 2.19 & 7.33 & $f c c+b c c$ & {$[52]$} \\
\hline $\mathrm{CoCrFeNi}$ & $\left.\mathrm{Al}_{0.92} \mathrm{CoCrF}\right] \mathrm{eNi}$ & - & 2.03 & 3.58 & 1.1066 & 0.86 & 13.38 & -10.73 & 2.10 & 7.26 & $f c c+b c c$ & {$[52]$} \\
\hline CoCrFeNi & $\left.\mathrm{Al}_{1.06} \mathrm{CoCr}\right] \mathrm{FeNi}$ & - & 2.12 & 3.72 & 1.1065 & 0.89 & 13.38 & -11.27 & 1.98 & 7.14 & $f c c+b c c$ & {$[52]$} \\
\hline $\mathrm{CoCrFeNi}$ & $\left.\mathrm{Al}_{1.06} \mathrm{CoC}\right] \mathrm{rFeNi}$ & - & 2.12 & 3.73 & 1.1065 & 0.89 & 13.38 & -11.36 & 1.96 & 7.17 & $\mathrm{fcc}+\mathrm{bcc}$ & {$[52]$} \\
\hline
\end{tabular}


Table 1 (continued)

\begin{tabular}{|c|c|c|c|c|c|c|c|c|c|c|c|c|}
\hline Substrates & Coatings or areas & $\mu$ & $\varepsilon$ & $\delta$ & $\gamma$ & $\alpha$ & $\Delta S_{\text {mix }}$ & $\Delta H_{\text {mix }}$ & $\Omega$ & VEC & Phase & References \\
\hline $1100 \mathrm{Al}$ & $\mathrm{AlCrFeC}] \mathrm{oNi}$ & 3.89 & 3.33 & 5.88 & 1.1705 & 1.21 & 11.58 & -8.06 & 2.43 & 6.68 & $\mathrm{fcc}+\mathrm{bcc}$ & [59] \\
\hline $45 \#$ steel & $\left.\mathrm{Al}_{2.0} \mathrm{CrF}\right] \mathrm{eCoCuNi}$ (upper region)] & 1.76 & 3.28 & 5.69 & 1.1693 & 1.26 & 14.85 & -5.26 & 4.53 & 7.59 & $\mathrm{fcc}+\mathrm{bcc}$ & {$[60]$} \\
\hline $45 \#$ steel & $\left.\mathrm{Al}_{2.0} \mathrm{CrF}\right] \mathrm{eCoCuNi}$ (central region)] & 1.76 & 3.09 & 5.39 & 1.1696 & 1.19 & 14.80 & -3.93 & 6.13 & 7.73 & $\mathrm{fcc}+\mathrm{bcc}$ & {$[60]$} \\
\hline AZ91 Mg & $\mathrm{AlCoCrCuFeNi}$ & 1.32 & 3.11 & 5.42 & 1.1686 & 1.22 & 14.82 & -5.91 & 4.09 & 7.63 & bcc & {$[61]$} \\
\hline Q235 & $\mathrm{CoCrFeAlCu}$ & 3.54 & 3.22 & 5.58 & 1.1692 & 1.32 & 13.38 & -1.02 & 20.97 & 7.47 & bcc & {$[62]$} \\
\hline Q235 & $\mathrm{FeCoCrAlCu}(\mathrm{ID})$ & 3.54 & 2.33 & 4.07 & 1.1697 & 0.89 & 11.03 & 6.36 & 2.62 & 9.01 & bcc & {$[63]$} \\
\hline Q235 & $\mathrm{FeCoCrAlCu}(\mathrm{DR})$ & 3.54 & 2.59 & 4.57 & 1.1704 & 0.96 & 12.47 & -1.74 & 12.34 & 7.57 & bcc & {$[63]$} \\
\hline Fe-Armco & TiZrNbMoV(300 W) & - & 4.51 & 6.97 & 1.2404 & 1.42 & 13.25 & -2.18 & 14.35 & 4.71 & bcc & {$[64]$} \\
\hline Fe-Armco & TiZrNbMoV(1000 W) & - & 4.82 & 7.78 & 1.2453 & 1.55 & 13.05 & -2.32 & 12.61 & 4.58 & bcc & {$[64]$} \\
\hline Q235 steel & MoFeCrTiWAl & 8.49 & - & 6.00 & - & - & 14.90 & -12.56 & 2.70 & 5.50 & bcc & {$[65]$} \\
\hline $45 \#$ steel & $\mathrm{FeCrCoNi}(\mathrm{SiC}-0)$ & 6.79 & 9.32 & 19.32 & 1.5551 & 2.94 & 11.82 & -27.68 & 0.92 & 5.98 & $\mathrm{fcc}+\mathrm{Cr}_{2} \mathrm{~B}$ & {$[55]$} \\
\hline $45 \#$ steel & $\mathrm{FeCrCoNi}(\mathrm{SiC}-0-900)$ & 6.79 & 9.30 & 19.26 & 1.5547 & 3.03 & 12.01 & -27.33 & 0.94 & 6.05 & $\mathrm{fcc}+\mathrm{Fe}_{2} \mathrm{~B}$ & {$[55]$} \\
\hline 45\# steel & $\mathrm{FeCrCoNiCBSi(SiC-5)}$ & 6.79 & 9.89 & 21.31 & 1.6637 & 3.50 & 13.81 & -38.48 & 0.85 & 5.64 & $\mathrm{fcc}+\mathrm{Fe}_{2} \mathrm{~B}$ & {$[55]$} \\
\hline $45 \#$ steel & $\mathrm{FeCrCoNiCBSi(SiC-5)}$ & 6.79 & 10.03 & 20.64 & 1.6477 & 3.88 & 12.49 & -48.22 & 0.66 & 6.12 & $\mathrm{fcc}+\mathrm{M}_{7} \mathrm{C}_{3}$ & {$[55]$} \\
\hline $45 \#$ steel & FeCrCoNiCBSi(SiC-5-900) & 6.79 & 9.64 & 20.40 & 1.6573 & 3.33 & 13.09 & -33.33 & 0.88 & 5.73 & $\mathrm{fcc}+\mathrm{Fe}_{2} \mathrm{~B}$ & {$[55]$} \\
\hline $45 \#$ steel & $\mathrm{FeCrCoNiCBSi}(\mathrm{SiC}-5-900)$ & 6.79 & 10.21 & 21.85 & 1.6613 & 3.64 & 13.31 & -46.93 & 0.72 & 5.75 & $\mathrm{fcc}+\mathrm{M}_{7} \mathrm{C}_{3}$ & {$[55]$} \\
\hline $45 \#$ steel & $\mathrm{FeCrCoNiCBSi}(\mathrm{SiC}-10)$ & 6.79 & 10.02 & 22.85 & 1.684 & 4.00 & 12.96 & -43.26 & 0.80 & 5.34 & $\mathrm{fcc}+\mathrm{Fe}_{2} \mathrm{~B}$ & {$[55]$} \\
\hline $45 \#$ steel & FeCrCoNiCBSi(SiC-10) & 6.79 & 10.37 & 22.04 & 1.659 & 3.31 & 12.02 & -51.30 & 0.62 & 5.90 & $\mathrm{fcc}+\mathrm{M}_{7} \mathrm{C}_{3}$ & {$[55]$} \\
\hline 45\# steel & FeCrCoNiCBSi(SiC-10-900) & 6.79 & 9.96 & 22.40 & 1.6791 & 3.70 & 12.99 & -40.31 & 0.81 & 5.42 & $\mathrm{fcc}+\mathrm{Fe}_{2} \mathrm{~B}$ & {$[55]$} \\
\hline 45\# steel & FeCrCoNiCBSi(SiC-10-900) & 6.79 & 10.34 & 22.79 & 1.6719 & 3.10 & 12.75 & -48.54 & 0.70 & 5.59 & $\mathrm{fcc}+\mathrm{M}_{7} \mathrm{C}_{3}$ & {$[55]$} \\
\hline 45\# steel & $\mathrm{FeCoCr}_{0.5} \mathrm{NiB}$ & 8.15 & 9.41 & 19.76 & 1.5587 & 2.77 & 12.02 & -27.02 & 0.94 & 5.86 & $\mathrm{fcc}+\mathrm{M}_{2} \mathrm{~B}$ & {$[66]$} \\
\hline 45\# steel & $\mathrm{FeCoCrNiB}$ & 8.15 & 9.43 & 19.79 & 1.5587 & 2.68 & 11.72 & -28.06 & 0.90 & 5.61 & $\mathrm{fcc}+\mathrm{M}_{2} \mathrm{~B}$ & {$[66]$} \\
\hline 45\# steel & $\mathrm{FeCoCr}_{1.5} \mathrm{NiB}$ & 8.15 & 9.48 & 20.01 & 1.5603 & 2.20 & 10.66 & -29.07 & 0.80 & 5.27 & $\mathrm{fcc}+\mathrm{M}_{2} \mathrm{~B}$ & {$[66]$} \\
\hline $45 \#$ steel & $\mathrm{FeCoCr}_{2.0} \mathrm{NiB}$ & 8.15 & 9.49 & 20.00 & 1.5610 & 1.93 & 9.89 & -29.52 & 0.74 & 5.12 & $\mathrm{fcc}+\mathrm{M}_{2} \mathrm{~B}$ & {$[66]$} \\
\hline $45 \#$ steel & $\mathrm{FeCoCr}_{3.0} \mathrm{NiB}$ & 8.15 & 9.46 & 19.87 & 1.5587 & 1.73 & 9.07 & -29.67 & 0.68 & 5.03 & $\mathrm{fcc}+\mathrm{M}_{2} \mathrm{~B}$ & {$[66]$} \\
\hline Q235 steel & $\mathrm{FeCoCrNiB}_{0.5}(\mathrm{~B})$ & 3.54 & 6.44 & 12.11 & 1.5231 & 2.80 & 12.93 & -14.58 & 1.73 & 7.35 & $\mathrm{fcc}+\mathrm{M}_{3} \mathrm{~B}$ & [67] \\
\hline Q235 steel & $\mathrm{FeCoCrNiB}_{0.75}(\mathrm{~B})$ & 3.54 & 5.98 & 11.16 & 1.5211 & 2.51 & 12.9 & -12.86 & 1.93 & 7.58 & $\mathrm{fcc}+\mathrm{M}_{3} \mathrm{~B}$ & {$[67]$} \\
\hline Q235 steel & $\mathrm{FeCoCrNiB}(\mathrm{B})$ & 3.54 & 6.19 & 11.58 & 1.5221 & 2.62 & 12.72 & -13.35 & 1.84 & 7.54 & $f c c+M_{3} B$ & {$[67]$} \\
\hline Q235 steel & $\mathrm{FeCoCrNiB}(\mathrm{C})$ & 3.54 & 6.41 & 12.05 & 1.523 & 2.71 & 12.19 & -14.53 & 1.66 & 7.02 & $\mathrm{fcc}+\mathrm{M}_{3} \mathrm{~B}$ & {$[67]$} \\
\hline Q235 steel & $\mathrm{FeCoCrNiB}_{1.25}(\mathrm{~B})$ & 3.54 & 6.05 & 11.31 & 1.5215 & 2.53 & 12.65 & -12.30 & 1.94 & 7.96 & $\mathrm{fcc}+\mathrm{M}_{3} \mathrm{~B}$ & {$[67]$} \\
\hline Q235 steel & $\mathrm{FeCoCrNiB}_{1.25}(\mathrm{C})$ & 3.54 & 6.65 & 12.54 & 1.5241 & 2.83 & 12.06 & -15.63 & 1.55 & 6.83 & $\mathrm{fcc}+\mathrm{M}_{3} \mathrm{~B}$ & {$[67]$} \\
\hline $45 \#$ steel & $\mathrm{FeCrNiCoMnB}_{0.75}$ & 2.36 & 9.23 & 18.95 & 1.5538 & 3.06 & 9.91 & -8.94 & 2.35 & 5.96 & $\mathrm{fcc}+\mathrm{M}_{2} \mathrm{~B}$ & {$[54]$} \\
\hline $45 \#$ steel & FeCrNiCoMnB & 2.36 & 9.25 & 19.08 & 1.5550 & 3.06 & 10.06 & -11.39 & 1.86 & 5.95 & $\mathrm{fcc}+\mathrm{M}_{2} \mathrm{~B}$ & {$[54]$} \\
\hline Q235 & $\mathrm{FeCoCrNiB}$ & 5.10 & 8.17 & 15.99 & 1.5346 & 3.69 & 13.22 & -21.37 & 1.24 & 6.89 & $\mathrm{fcc}+\mathrm{M}_{3} \mathrm{~B}$ & {$[68]$} \\
\hline Q235 & $\mathrm{FeCoCrNiB}_{0.5}$ & 3.54 & - & 8.00 & - & - & 13.15 & -13.30 & 1.84 & 7.70 & $\mathrm{fcc}+\mathrm{M}_{2} \mathrm{~B}$ & {$[70]$} \\
\hline Q235 & MoFeCrTiW & 8.49 & - & 6.10 & - & - & 13.38 & -5.76 & 5.80 & 6.00 & $\mathrm{bcc}+\mathrm{IM}$ & {$[65]$} \\
\hline Q235 & MoFeCrTiWSi & 8.49 & - & 7.50 & - & - & 14.90 & -24.89 & 1.40 & 5.70 & $\mathrm{bcc}+\mathrm{IM}$ & {$[65]$} \\
\hline Q235 & MoFeCrTiWSiAl & 8.49 & - & 7.30 & - & - & 16.18 & -24.57 & 1.40 & 5.30 & $\mathrm{bcc}+\mathrm{IM}$ & {$[65]$} \\
\hline
\end{tabular}

The inter-dendritic area in the multi-component alloy coatings is abbreviated by ID, and the area of dendritic is abbreviated to DR. In Ref. [55], the number of 0 in SiC-0-900 indicates the amount (mole percent) of additives of $\mathrm{SiC}$ and the number of 900 (centigrade) indicates the annealing temperature 
Then the normalized parameter $\gamma$ of the geometric packing state to reveal the atomic packing instability is brought forward:

$\gamma=\frac{\omega_{S}}{\omega_{L}}=\frac{1-\sqrt{\frac{\left(r_{S}+\bar{r}\right)^{2}-\vec{r}^{2}}{\left(r_{S}+\bar{r}\right)^{2}}}}{1-\sqrt{\frac{\left(r_{L}+\bar{r}\right)^{2}-\vec{r}^{2}}{\left(r_{S}+\bar{r}\right)^{2}}}}$.

However, this criterion of $\gamma$ is not completely applicable to the HEA coatings as the element contents cannot be assured coincident. Particularly, the effects of the trace elements (such as $\mathrm{C}, \mathrm{S}, \mathrm{N}, \mathrm{Si}$ ) which originated from the substrate should not be overestimated even if their atomic sizes are significantly different from the others. With this in mind, only the elements whose actual contents exceed ten percent in the coatings are considered in the calculation of $\gamma$, and the data are listed in Table 1.

Besides, considering the lattice distortion is closely related to the atomic size difference in an alloy system [77-79], a phenomenological model of local lattice distortions in HEAs was proposed to reveal the effects of the atomic size difference on phase stability [25]. The local lattice distortions were calculated by the comparison of one distorted lattice and its ideal counterpart lattice, in which the ideal lattice was treated as the reference state and then the dimensionless displacement of one atom that stems from the ideal atoms was denoted by the formula of $\left|r_{i}-\bar{r}\right| / r$. Therefore, the total displacement of the HEAs is given as [25]:

$\alpha_{1}=\sum_{i=1}^{n} c_{i} \frac{\left|r_{i}-\bar{r}\right|}{\bar{r}}$

Moreover, it should be noted that the local lattice distortion cannot be clearly expressed just by one single atom. On the other hand, the adjacent atoms in an alloy system reflect the local lattice distortion more accurately, where the dimensionless displacement for an atomic and its counterpart atomic pair is defined as $\left|r_{i}+\bar{r}_{j}-2 \bar{r}\right| / 2 \bar{r}$.. By considering this point, the parameter to reflect the local lattice distortion of HEAs is given as follows [25]:

$\alpha_{2}=\sum_{i=1}^{n} \sum_{j>i}^{n} c_{i} c_{j} \frac{\left|r_{i}+r_{j}-2 \bar{r}\right|}{2 r}$.

\subsection{Factors of Thermodynamic}

Near equimolar ratios for HEAs inevitably lead to an increase in the alloy system chaos, and then the formation ability of unfavorable intermetallic compounds is weakened $[26,80]$. By contrast, the formation of the simple solid solution phases such as FCC and BCC tends towards simplicity owing to the randomly located atoms in the crystal lattice. Based on this, there may be a definite range of mixing entropy to prognosticate the formation ability of the solid solution phases in HEAs. Being based upon the Boltzmann's hypotheses of the entropy, the calculation method of mixing entropy for an n-component alloy is proposed as the follows $[23,26]$ :

$\Delta S_{\text {mix }}=-R \sum_{i=1}^{n} c_{i} \ln c_{i}$

where $R$ represents the molar gas constant $\left(R=1.38 \times 10^{13} \mathrm{~J} / \mathrm{K}\right)$

System temperature is the derivative of internal energy to mixing entropy according to the physical definition, and it is undeniable that more quantity of heat is absorbed by the alloy system when the melting point of elements is relatively higher. Then considering the definition of HEAs (consisted of at least five elements), the effects of the melting points on the mixing entropy must be more significant. Therefore, synthetically considering the two factors (melting point, mixing entropy) is very meaningful for the study of solid solution stability in laser-clad HEA coatings. Therefore, comprehensive parameter $T_{\mathrm{m}} \Delta S_{\text {mix }}$ was quoted [26], in which the melting point of HEA systems is denoted by $T_{\mathrm{m}}$, which is as follows:

$T_{m}=\sum_{i=1}^{n} c_{i}\left(T_{m}\right)_{i}$

where $\left(T_{\mathrm{m}}\right)_{i}$ represents the melting point of the $i$ th element.

Based on the achievements that have been acknowledged, the formula of Gibbs free energy $(G)$ for multi-component HEAs is defined as [23]:

$\Delta G_{\text {mix }}=\Delta H_{\text {mix }}-T \Delta S_{\text {mix }}$.

Relatively negative values of $\Delta H_{\text {mix }}$ are beneficial for the formation of chemically ordered phases, which denotes that the formation ability of intermetallic compounds is favored but not the simple solid solution. And the atoms of an alloy system are tended to be mutually exclusive when the values of $\Delta H_{\text {mix }}$ are much too positive, which increase the segregated possibility for the elements. Therefore, the Miedema model which is known as the thermodynamic model to calculate the mixing enthalpy $\left(\Delta H_{\text {mix }}^{A B}\right)$ for binary alloy systems was cited to probe the influences of $\Delta H_{\text {mix }}$ on the formation ability of solid solution phases for HEAs [81]:

$\Delta H_{\text {mix }},=\sum_{i, j=1, i \neq j}^{n} c_{i} c_{j} \Omega_{i, j}$. 
where $\Omega_{i, j}$ is denoted the interaction parameter of the $i$ th and $j$ th elements (calculated by $4 \Delta H_{\text {mix }}^{A B}$ ).

At present, Wang et al. [26] put forward a significant conclusion that the effects of $T_{\mathrm{m}} \Delta S_{\text {mix }}$ and $\mathrm{I} \Delta H_{\text {mix }}$ I were opposite on the formation of solid solution phases. The larger values of $T_{\mathrm{m}} \Delta S_{\text {mix }}$ provided driving power for the formation of solid solution phases, and the phases stability was inhibited when the absolute values of $\Delta H_{\text {mix }}$ are relatively higher. To overall evaluate the effects of the two factors $\left(T_{\mathrm{m}} \Delta S_{\text {mix }}\right.$ and $\left.\left|\Delta H_{\text {mix }}\right|\right)$ on the phase stability, parameter $\Omega$ also has been cited, and the formula is defined as [26]:

$\Omega=\frac{T_{m} \Delta S_{\mathrm{mix}}}{\left|\Delta H_{\mathrm{mix}}\right|}$.

\subsection{Factors of Valence Electron Concentration}

It not only requires to distinguish the formation ability of solid solution phases and intermetallic compounds, but the alloys composed of different solid solution phases also possess diverse performances and characteristics. For instance, the hardness for the alloys composed of BCC or HCP phases is generally higher than it is of FCC due to the slip systems is relatively less in FCC. And the plastic performance of FCC is lower than BCC owing to the correspondingly higher stacking fault energy for FCC. Therefore, exploring the criteria to predict the formation ability of different kinds of solid solution phases for HEAs is a meaningful work. In relevant studies, Guo et al. [27, 28] proposed that the sole phase of FCC in HEAs is stable when the values of valence electron concentration (VEC) are at a relatively larger range, while the reasonably smaller values of VEC is favor to form of sole BCC phase. The calculation formula of VEC is given as follows [27]:

$\mathrm{VEC}=\sum_{i=1}^{n} c_{i}(\mathrm{VEC})_{i}$

in which (VEC) $)_{i}$ and $c_{i}$ refer to the value of VEC and the atomic percent of the $i$ th element, respectively.

\section{Criteria and Discussion}

The statistical results of all the parameters corresponding to different phase compositions for laser-clad HEA coatings are listed in Table 1. Based on the analyses of considerable data gained from the publicly reported references related to the laser-clad HEAs coatings, the effects of process parameters $(\mu)$, dynamics $(\varepsilon, \delta, \gamma, \alpha)$, thermodynamic $\left(\Delta S_{\text {mix }}, \Delta H_{\text {mix }}, \Omega\right)$ and the valence electron concentration (VEC) on the phase stability for laser-clad HEA coatings are proposed in this study.

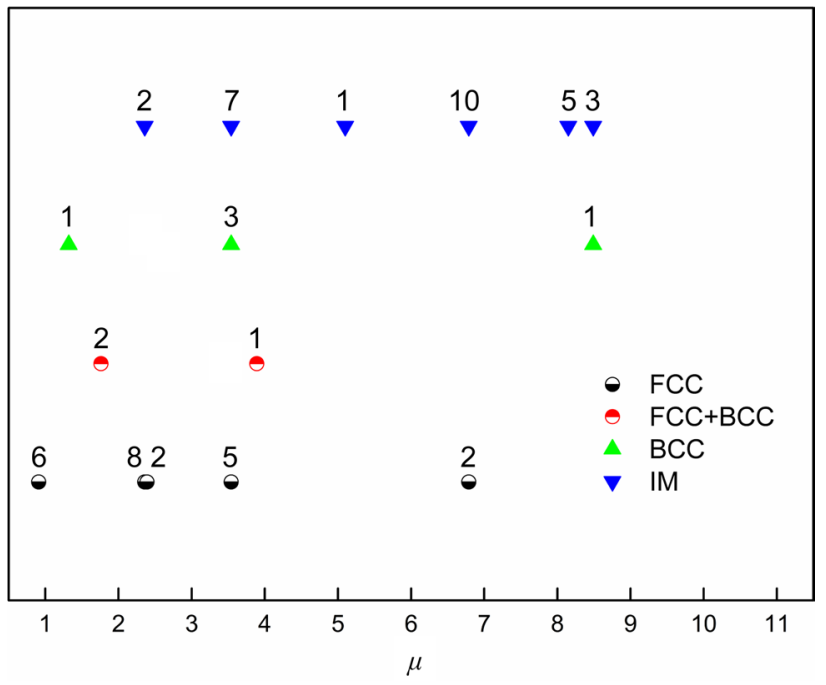

Fig. 1 Effects of $\mu$ on the phase stability for HEA coatings fabricated by laser cladding process. The digits on the identification point mean the number of occurrences of the corresponding phases

\subsection{Criterion of Process Parameters}

Figure 1 presents the effects of process parameters on the phase stability for laser-clad HEA coatings. More than $90 \%$ of the solid solution phases are obtained at the range of $\mu \leq 4$, nevertheless, nearly $70 \%$ of the intermetallic phases are synthesized when $\mu>4$, which denotes that a relatively lower laser density is advantageous to the formation of solid solution phases for laser-clad HEA coatings. It is well consistent with the previous analysis that the melting amount of the substrate part is increased seriously when the laser energy density is much too high $(\mu>4)$, then the element's content of the substrate exceeded the expectation in the eventually HEA coatings. Besides, the cooling rate also is weakened with along heat transfer distance when the depth of the melted substrate part becomes larger, then a great amount of energy can be accumulated in the molten pool. Therefore, $\mu \leq 4$ is identified as the process criterion for the preparation of laser-clad HEA coatings which consisted of solid solution phases.

\subsection{Criteria of the Atomic Size Difference}

Figure 2 a presents the effects of the atomic size difference $(\varepsilon)$ on the phase formation for laser-clad HEA coatings. The coatings composed of solid solution phases all distributed at the regions with a relatively lower atomic size difference, which is well inconformity with the Hume-Rothery rules [22] and the discussion given in the previous chapters. And it can be concluded that the maximum range of $\varepsilon$ for solid solution phases is 5.72 , while the minimum range for the formation of intermetallic phases is 5.98 based on the data 

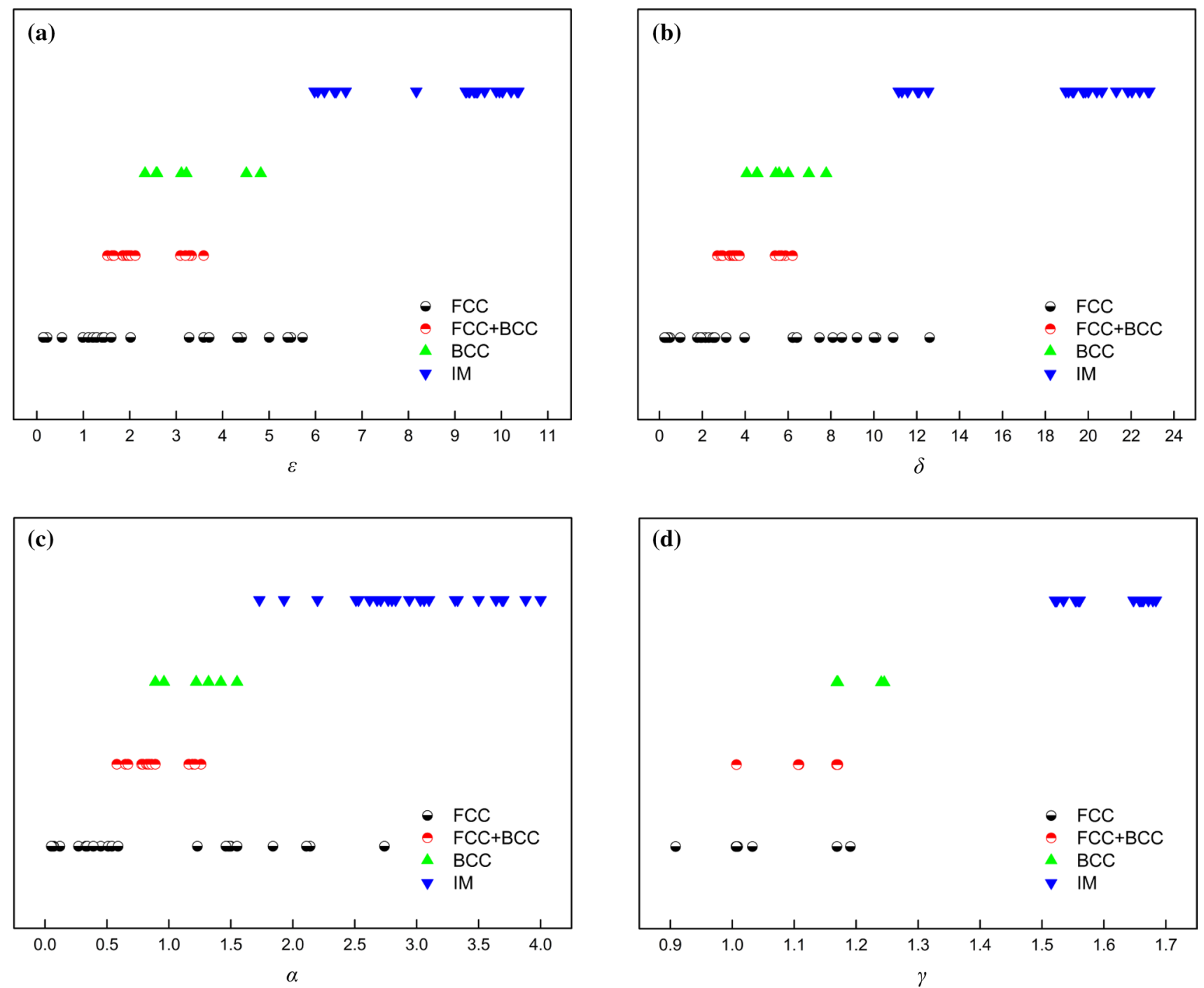

Fig. 2 Effects of atomic size difference on the phase stability for HEA coatings fabricated by laser cladding process: a effects of $\varepsilon$ on the phase stability; $\mathbf{b}$ effects of $\delta$ on the phase stability; $\mathbf{c}$ effects of $\alpha$ on the phase stability; $\mathbf{d}$ effects of $\gamma$ on the phase stability

analysis. It is revealed that the formation ability of solid solution phases and intermetallic phases can be almost completely distinguished by $\varepsilon$, and a criterion to predict the phase stability is proposed that the HEA coatings prepared by laser cladding process is tend to be consisted of solid solution phases when $\varepsilon \leq 5.8$.

In the other works, parameter $\delta$ also has been applied to evaluate the effects of atomic size difference on the phase stability for HEAs. Guo et al. [28] proved that $0 \leq \delta \leq 8.5$ is one of the criteria to predict the formation of solid solution phases. Besides, $\delta \leq 6.6$ [26] also has been recognized to apply to the solid solution phases stability criterion for HEAs. However, Ren et al.'s [82] found that only the systems satisfied $\delta \leq 2.77$, and the HEAs are composed of simple solid solutions. Although some conclusive results have been obtained in the other works, there is still no completely unified and precise criterion about $\delta$ in the prediction of solid solution stability for HEAs. Figure $2 \mathrm{~b}$ shows the values of $\delta$ corresponding to different phase compositions for laserclad HEA coatings. It is clear that the overlap for solid solution phases and intermetallic phases is not serious, which denotes that $\delta$ is a reliable criterion in the prediction of the phase formation ability for laser-clad HEA coatings. And the data analysis shows that almost all of the solid solution phases are located at the area of $\delta>11$, and more than $80 \%$ of the laser-clad HEA coatings which consisted of intermetallic compounds are synthesized at the opposite range $(\delta>11)$. Meanwhile, it can be concluded that a relatively smaller atomic size difference is in favor of the formation of solid solution phases for laser-clad HEA coatings, which is well agreed with the analysis results of the atomic size difference criterion $\varepsilon$. 
Figure 2c shows the effects of the adjacent atoms' atomic size difference on the phase stability for laser-clad HEA coatings. The results indicate that about $95 \%$ of the solid solution phase HEA coatings are synthesized at the region of $\alpha \leq 2$, while $92 \%$ of the intermetallic coatings are formed when $\alpha>2$, which signified that the phases stability of laserclad HEA coatings are estimated by the lattice-distortion criterion $\alpha$. Therefore, $\alpha \leq 2$ is utilized as a criterion to prognosticate the formation ability of solid solution phases for laser-clad HEA coatings. This illustrates that serious lattice distortion is beneficial for the formation of intermetallic phases, and relatively lower lattice distortion is conducive to the formation of solid solution phases.

Figure $2 d$ shows the effects of the specific size elements on the phase stability for laser-clad HEA coatings, where the atomic packing instability is revealed by the normalized parameter. And it should be pointed out that only the elements whose actual content exceeds ten percent in the coatings were allowed to the calculation of $\gamma$ to avoid the impact of the trace elements (such as C, S, N, Si) which originated from the substrate. Then a significant phenomenon can be observed that the coatings composed of solid solution phases all synthesized at a lower range of $\gamma(\gamma \leq 1.24)$, and the forming ability of intermetallic phases is promoted when the atomic packing instability is relatively serious $(\gamma \geq 1.52)$. This means that a relatively smaller disparity of the smallestsize and largest-size atoms is favorable to the formation of solid solution phases for laser-clad HEA coatings. However, there is still a pronounced range of $\gamma(1.24<\gamma<1.52)$ where the phases stability cannot be distinguished clearly, which denotes that the atomic packing parameter $\gamma$ is not competent

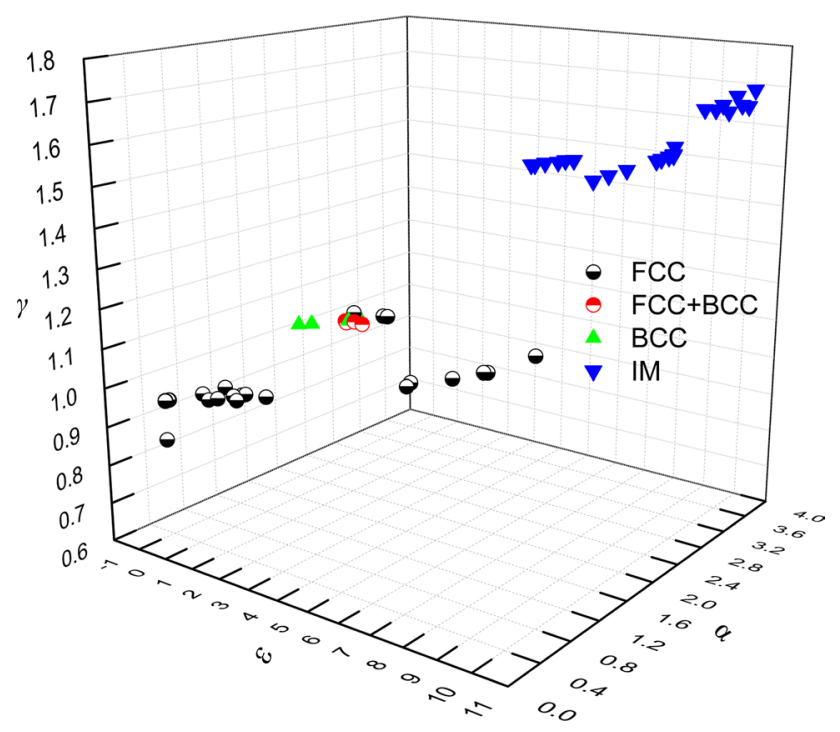

Fig. 3 Superposed effects of $\varepsilon, \gamma$ and $\alpha$ on the phase stability for HEA coatings fabricated by laser cladding process enough to estimate the potential possibility of the phase formation for laser-clad HEA coatings.

The analyses about $\varepsilon, \delta$ and $\alpha$ indicate that the formation of solid solution phases is promoted when the atomic size difference is relatively small, as shown in Fig. 3, and the criteria of $\varepsilon \leq 5.8, \delta \leq 11$ and $\alpha \leq 2$ can be used to prognosticate the formation ability of solid solution phases for laser-clad HEA coatings.

\subsection{Criteria of Thermodynamic}

Figure $4 \mathrm{a}$ shows the data distribution of $\Delta S_{\mathrm{mix}}$ corresponding to the formation of different phases for laserclad HEA coatings. The values of $\Delta S_{\text {mix }}$ which consisting of solid solution phases are almost greater than 10.8, while it is more concentrated at the range of $\Delta S_{\text {mix }}<13.5$ for intermetallic phases. It is indicated that the higher values of $\Delta S_{\text {mix }}$ are conducive to the formation of solid solution phases for laser-clad HEA coatings. However, it also can be found that there is an obvious overlapping part $\left(11.5<\Delta S_{\text {mix }}<13.5\right)$ of $\Delta S_{\text {mix }}$ corresponding to intermetallic and solid solution phases, which means that $\Delta S_{\text {mix }}$ as a criterion to predict the formation ability of solid solution phases for laser-clad HEA coatings is not accurate enough. Meanwhile, it should be noted that the values of $\Delta S_{\text {mix }}$ for the laser-clad HEA coatings is obviously higher for both intermetallic phase $\left(\Delta S_{\mathrm{mix}}>9.5\right)$ and solid solution phase $\left(\Delta S_{\operatorname{mix}}>10.5\right)$ when compared with that of the binary alloys $\left(\Delta S_{\text {mix }}=5.74\right.$ when $\left.n=2\right)$ due to HEAs are defined to consist of at least five principal elements. Figure $4 \mathrm{~b}$ presents the effects of $\Delta H_{\text {mix }}$ on the phase formation for laser-clad HEA coatings, where almost all of the coatings consisted of solid solution phases are formed at the range of $-14.5 \leq \Delta H_{\text {mix }} \leq 6.5$, and more than $70 \%$ intermetallic-composed coatings have existed when $\Delta H_{\text {mix }}<-14.5$. It indicates that $-14.5 \leq \Delta H_{\text {mix }} \leq 6.5$ is an appropriate criterion to predict the solid solution phases formation for laser-clad HEA coatings.

Then based on the analysis of $\Delta S_{\text {mix }}$ and $\Delta H_{\text {mix }}$, the parameter $\Omega$ is cited to comprehensively evaluate the influence of the thermodynamic factors on the phase stability of laser-clad HEA coatings. The results shown in Fig. 4c demonstrate that more than $93 \%$ of the laser-clad HEA coatings which consisted of solid solution phases are obtained under the conditions of $\Omega \geq 1.8$, and nearly $80 \%$ coatings that composed of intermetallic phases are synthesized when $\Omega$ is less than 1.8. This means that the dynamic force (denoted by $T_{\mathrm{m}} \Delta S_{\mathrm{mix}}$ ) of solid solution phases formation for laser-clad HEA coatings is just equal to the resistance force (denoted by $\left|\Delta H_{\text {mix }}\right|$ ) when $\Omega=1.8$ is satisfied. It proves 

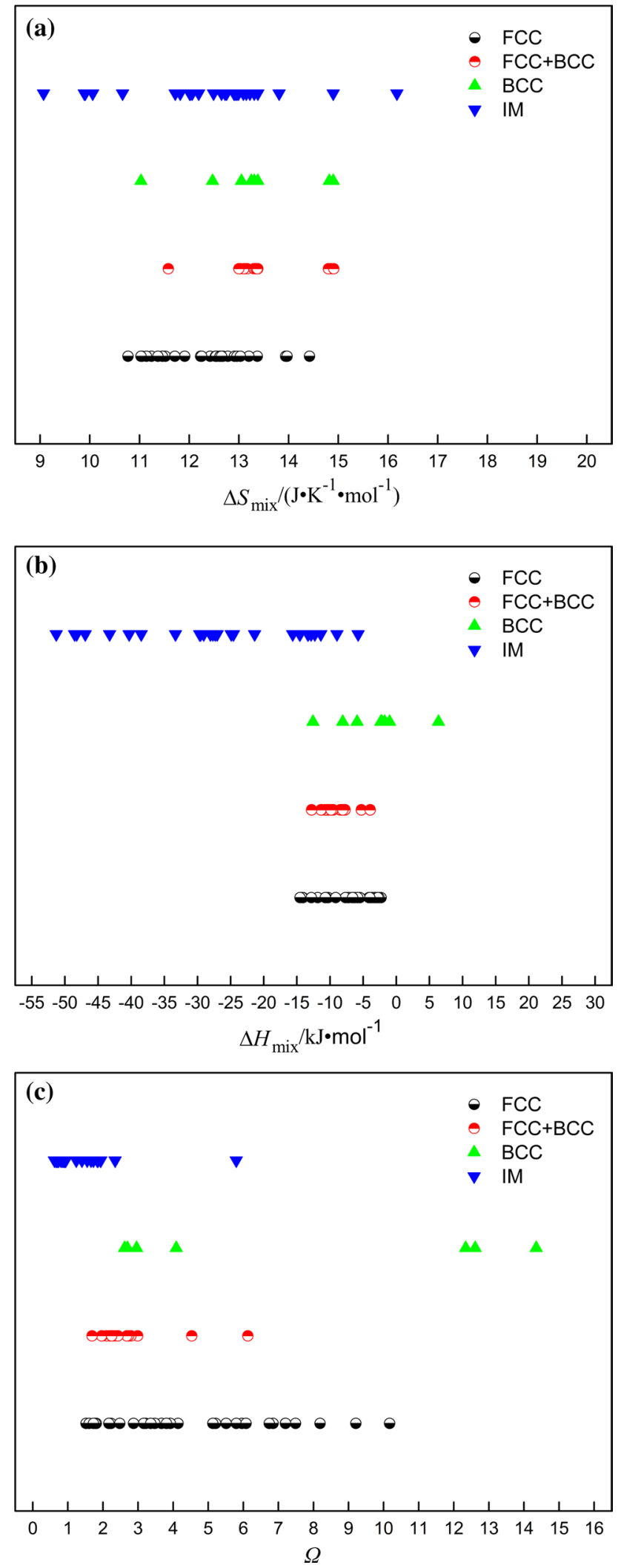

Fig. 4 Effects of thermodynamic on the phase stability for HEA coatings fabricated by laser cladding process: a effects of $\Delta S_{\text {mix }}$ on the phase stability; $\mathbf{b}$ effects of $\Delta H_{\text {mix }}$ on the phase stability; $\mathbf{c}$ effects of $\Omega$ on the phase stability

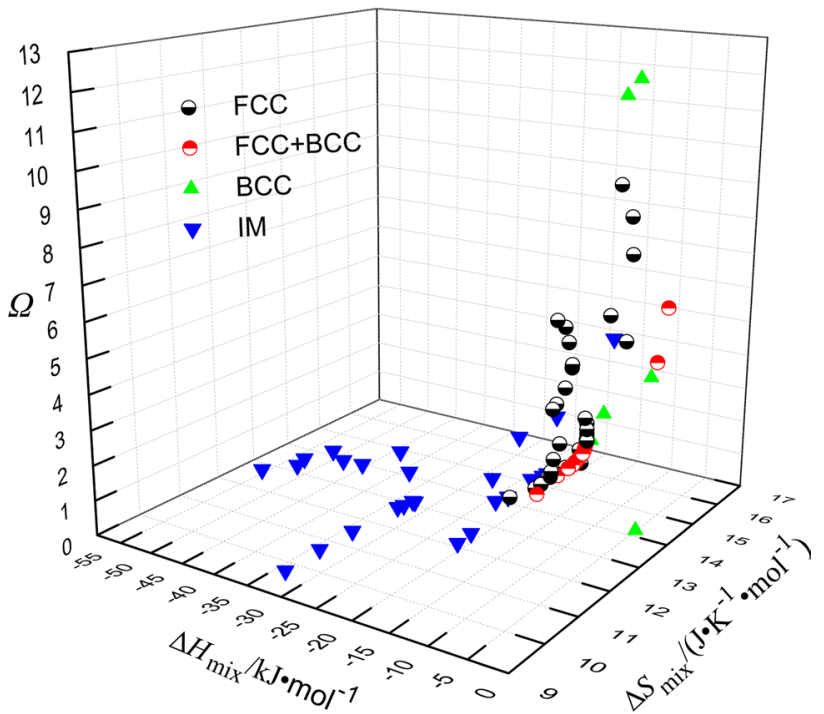

Fig. 5 Superposed effects of $\Delta S_{\text {mix }}, \Delta H_{\text {mix }}$ and $\Omega$ on the phase stability for HEA coatings fabricated by laser cladding process

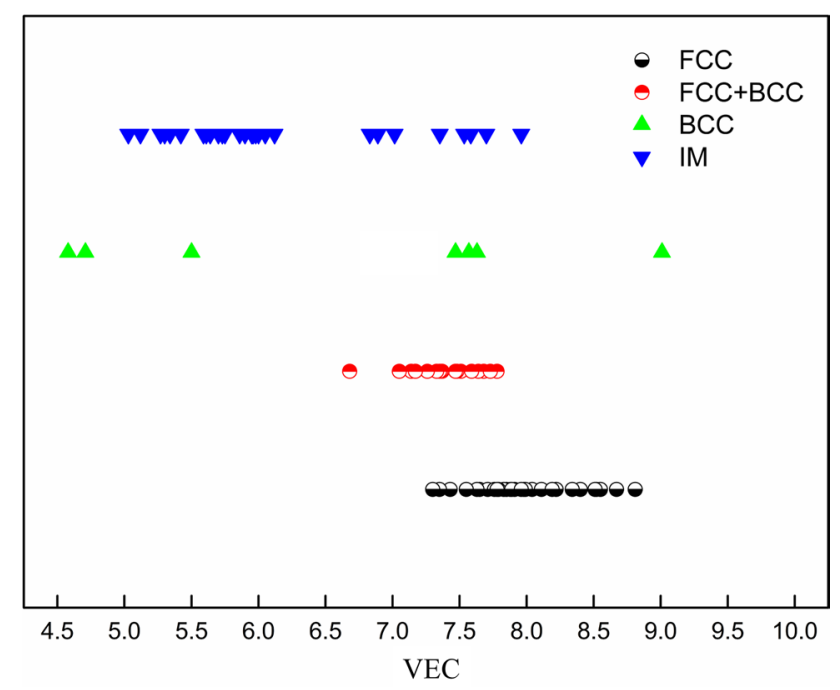

Fig. 6 Effects of VEC on the phase stability for HEA coatings fabricated by laser cladding process

that a relatively large degree of the alloy system chaos and a relatively small enthalpy of mixing are conducive to the formation of solid solution phases.

Consequently, it is feasible to take $-14.5 \leq \Delta H_{\text {mix }} \leq 6.5$ and $\Omega \geq 1.8$ as the thermodynamic criteria for the formation of solid solution phases in laser-clad HEA coatings with the comprehensive analysis of $\Delta S_{\text {mix }}, \Delta H_{\text {mix }}$ and $\Omega$ shown in Fig. 5. 


\subsection{Criterion of Valence Electron Concentration}

Figure 6 reflects the connection between valence electron concentration (VEC) and the phase stability of laser-clad HEA coating. A remarkable phenomenon can be found that the phase compositions of the HEA coatings gradually shift from BCC to dual-phase composed of BCC and FCC with the increase in VEC, and then a single FCC phase is obtained ultimately. Moreover, the calculation results show that the VEC values corresponding to $86 \%$ BBC HEA coatings are less than 7.65, while the VEC values for $73 \%$ FCC HEA coatings are greater than 7.65. Meanwhile, most of the dual-phase HEA coatings are prepared at the range of $7.14<$ VEC $<7.78$. Therefore, the criteria VEC for the solid solution phase formation of laser-clad HEA coatings are concluded as follows: VEC $<7.65$ for simple BCC phase, VEC $\geq 7.65$ for simple FCC phase, $7.14<$ VEC $<7.78$ for dual-phase BCC and FCC.

\subsection{Discussions and Comparisons of Criteria}

To better study the accuracy of the criteria on the phase prediction for laser-clad HEA coatings, further analyses are taken as shown in Figs. 7 and 8, in which the atomic size criterion $\varepsilon$ which distinguishes the phase stability most clearly is adopted as a reference coordinate system. It is obvious that the formation of solid solution phases for laser-clad HEA coatings requires the process parameters satisfy $\mu \leq 4$, atomic size difference satisfy $\varepsilon \leq 5.8, \delta \leq 11$ and $\alpha \leq 2$, thermodynamic criteria satisfy $-14.5 \leq \Delta H_{\text {mix }} \leq 6.5$ and $\Omega \geq 1.8$. In addition to the criteria $\mu$ and $\varepsilon$ proposed firstly, there are some differences for the other modified criteria $\left(\delta, \alpha, \Delta H_{\text {mix }}\right.$, $\Omega$, VEC) to compare with ones of the traditional bulk-body

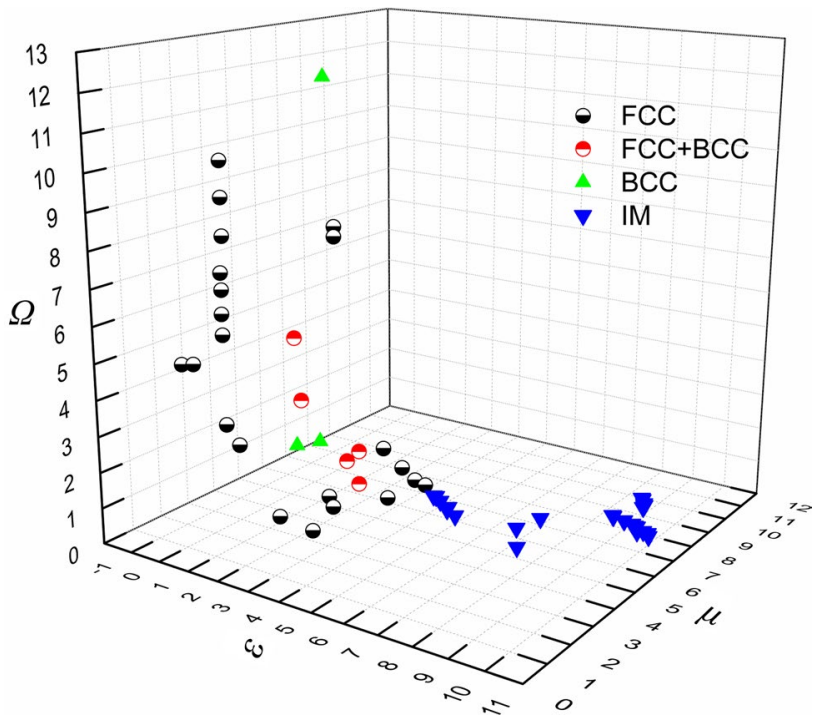

Fig. 8 Superposed effects of $\varepsilon, \mu$ and $\Omega$ on the phase stability for HEA coatings fabricated by laser cladding process

HEAs. The laser-clad HEA coatings composed of solid solution phases can be prepared with a greater scale of atomic size difference $(\delta \leq 11)$ to compete against the studies of Guo et al. $(\delta \leq 8.5)$ [28] and Yang et al. $(\delta \leq 6.6)$ [26]. This phenomenon is closely related to the rapid heating and the cooling characteristics for laser cladding process, where the long-range diffusion of the metal atoms is inhibited effectively. It restrains the nucleation and growth of the intermetallic phases, but the formation of the solid solution phases is promoted. Therefore, the requirement of the atomic size is relatively lower for laser cladding HEA coatings when compared with that of the traditional bulk-body HEAs.
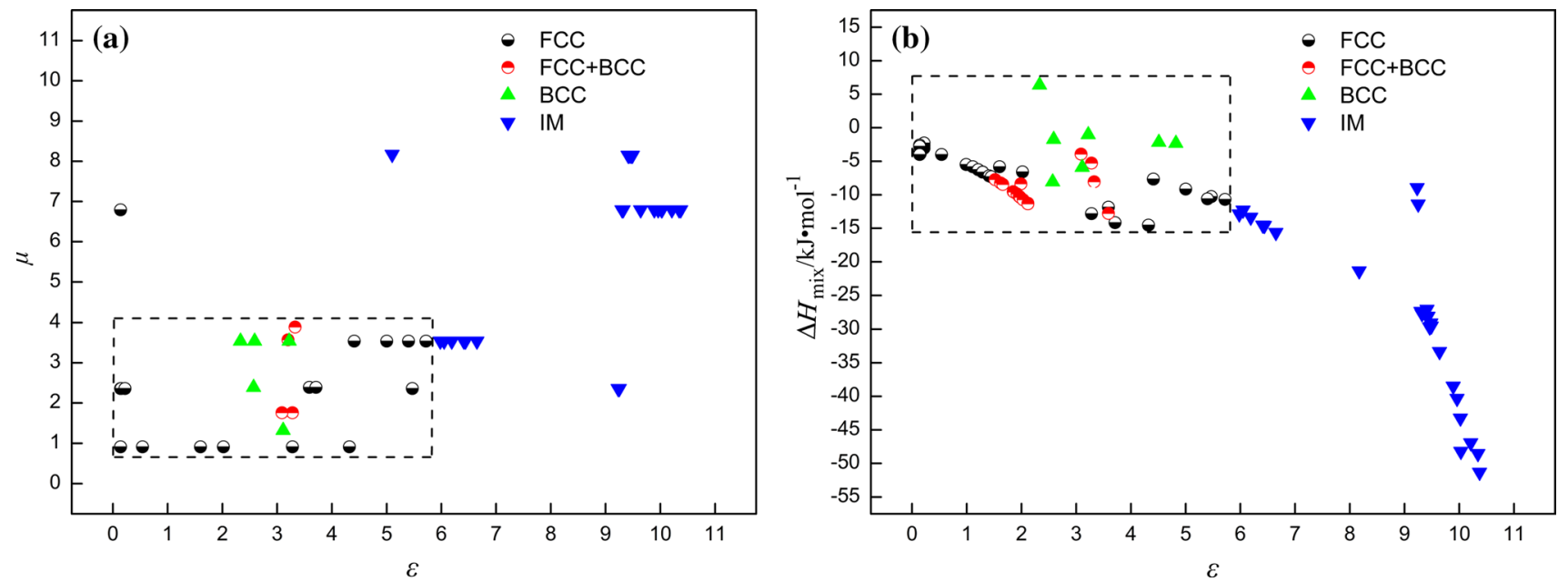

Fig. 7 Superposed effects of process parameters, atomic size difference and thermodynamic on the phase stability for HEA coatings fabricated by laser cladding process: a the superposed effects of $\varepsilon$ and $\mu$ on the phase stability; $\mathbf{b}$ the effects of $\varepsilon$ and $\Delta H_{\text {mix }}$ on the phase stability 


\section{Conclusions}

In this study, based on the previous works and considering the characteristics of rapid heating and cooling rates for laser cladding process, the criteria of process parameters $(\mu)$, atomic size difference $(\varepsilon, \delta, \gamma$ and $\alpha)$, thermodynamic $\left(\Delta S_{\text {mix }}, \Delta H_{\text {mix }}\right.$ and $\Omega$ ) and VEC are studied to predict the phase stability for laser-clad HEA coatings. The main conclusions are as follows:

(1) Relatively lower laser density is beneficial for the formation of solid solution phases and $\mu \leq 4$ has been proved to be the process parameters criterion in determining the formation of solid solution phases for laserclad HEA coatings.

(2) The criteria of atomic size difference $(\varepsilon, \delta, \gamma$ and $\alpha$ ) are consistent with the Hume-Rothery rules that a relatively small difference in atomic size is conducive to the formation of solid solution phases, in which $\varepsilon \leq 5.8$, $\delta \leq 11$ and $\alpha \leq 2$ are taken as the criteria to predict the formation of solid solution phases. The criterion $\varepsilon$ which proposed firstly separates the phases (intermetallic and solid solution phases) very accurately. The modified criteria of thermodynamic $-14.5 \leq \Delta H_{\text {mix }} \leq 6.5$ and $\Omega \geq 1.8$ are taken to estimate the formation ability of solid solution phases for laser-clad HEA coatings.

(3) Relatively large values of VEC are contributed to the phase formation of FCC for HEA coatings. The criteria of VEC to distinguish the formation ability of solid solution phases for laser-clad HEA coatings are shown as follows: $\mathrm{VEC}<7.65$ for simple $\mathrm{BCC}, \mathrm{VEC} \geq 7.65$ for simple FCC, $7.14<$ VEC $<7.78$ for dual-phase BCC and FCC.

Acknowledgement This work was financially supported by the National Natural Science Foundation of China (No. 51627802).

\section{References}

[1] J.W. Yeh, S.K. Chen, S.J. Lin, J.Y. Gan, T.S. Chin, T.T. Shun, Adv. Eng. Mater. 6, 299 (2004)

[2] A.M. Li, X.Y. Zhang, Acta Metall. Sin. -Engl. Lett. 22, 219 (2019)

[3] Z.M. Li, K.G. Pradeep, Y. Deng, D. Raabe, C.C. Tasan, Nature 534, 227 (2016)

[4] M. Wang, Z.M. Li, D. Raabe, In-situ Acta Mater. 147, 236 (2018)

[5] Q.H. Fang, Y. Chen, J. Lia, C. Jiang, B. Liu, Y. Liu, P.K. Liaw, Int. J. Plast. 114, 161 (2019)

[6] X.L. Shang, Z.J. Wang, Q.F. Wu, J.C. Wang, J.J. Li, Acta Metall. Sin. -Engl. Lett. 32, 41 (2019)

[7] D.B. Miracle, O.N. Senkov, Acta Mater. 122, 448 (2017)

[8] E.J. Pickering, N.G. Jones, Int. Mater. Rev. 61, 183 (2016)

[9] Y.T. Wang, J.B. Li, Y.C. Xin, X.H. Chen, M. Rashad, B. Liu, Y. Liu, Acta Metall. Sin. -Engl. Lett. 32, 932 (2019)
[10] B. Chanda, J. Das, J. Alloys Compd. 798, 167 (2019)

[11] D.X. Qiao, H. Jiang, W.N. Jiao, Y.P. Lu, Z.Q. Cao, T.J. Li, Acta Metall. Sin. -Engl. Lett. 32, 925 (2019)

[12] R.L. Carlyn, S. Mulaine, V. Céline, G. Maryam, Mater. Charact. 151, 310 (2019)

[13] Z.W. Tang, S. Zhang, R.P. Cai, Q. Zhou, H.F. Wang, Metall. Mater. Trans. A 50, 1888 (2019)

[14] Y.F. Juan, J. Li, Y.Q. Jiang, W.L. Jia, Z.J. Lu, Appl. Surf. Sci. 465, $700(2019)$

[15] Y.M. Tan, J.S. Li, S.W. Tang, J. Wang, H.C. Kou, J. Alloys Compd. 742, 430 (2018)

[16] E. Huang, C.M. Lin, J. Juang, Y.J. Chang, Y.W. Chang, C.S. Wu, C.W. Tsai, A.C. Yeh, S.R. Shieh, C.P. Wang, Y.C. Chuang, Y.F. Liao, D. Zhang, T. Huang, T.N. Lam, Y.H. Chen, J. Alloys Compd. 792, 116 (2019)

[17] C. Xiang, Z.M. Zhang, H.M. Fu, E.H. Han, J.Q. Wang, H.F. Zhang, G.D. Hu, Acta Metall. Sin. -Engl. Lett. 32, 1053 (2019)

[18] N. Ishizu, J. Kitagawa, Results Phys. 13, 102274 (2019)

[19] Y.Y. Zhao, H.W. Chen, Z.P. Lu, T.G. Nieh, Acta Mater. 147, 184 (2018)

[20] Y.F. Wang, S.G. Ma, X.H. Chen, J.Y. Shi, Y. Zhang, J.W. Qiao, Acta Metall. Sin. -Engl. Lett. 26, 277 (2019)

[21] B. Kombaiah, K. Jin, H. Bei, P.D. Edmondson, Y. Zhang, Mater. Des. 160, 1208 (2018)

[22] H. Jones, Proc. Phys. Soc. 49, 250 (2002)

[23] Y. Zhang, Y.J. Zhou, J.P. Lin, G.L. Chen, K.L. Peter, Adv. Eng. Mater. 10, 534 (2008)

[24] Z.J. Wang, Y.H. Huang, Y. Yang, J.C. Wang, C.T. Liu, Scr. Mater. 94, 28 (2015)

[25] Z.J. Wang, W.F. Qiu, Y. Yang, C.T. Liu, Intermetallics 64, 63 (2015)

[26] X. Yang, Y. Zhang, Mater. Chem. Phys. 132, 233 (2012)

[27] S. Guo, C.T. Liu, Prog. Nat. Sci.: Mater. Int. 21, 433 (2011)

[28] S. Guo, C. Ng, J. Lu, C.T. Liu, J. Appl. Phys. 109, 213 (2011)

[29] M.C. Troparevsky, R.M. James, M. Daene, Y. Wang, R.L. Andrew, M. Stocks, JOM. 67, 2350 (2015)

[30] W.M. Choi, Y.H. Jo, D.G. Kim, S.S. Sohn, S. Lee, B.J. Lee, J. Phase Equilib. Diffus. 39, 694 (2018)

[31] W.J. Huang, P. Martin, H.L. Zhuang, Acta Mater. 169, 225 (2019)

[32] Y. Tian, C.Y. Lu, Y.F. Shen, X.M. Feng, Surf. Interfaces 15, 135 (2019)

[33] G. Muvvala, D.P. Karmakar, A.K. Nath, Mater. Des. 121, 310 (2017)

[34] Q.H. Fang, Y. Chen, J. Li, Y.B. Liu, Y. Liu, Phys. B. 550, 112 (2018)

[35] S. Yin, W.Y. Li, B. Song, X.C. Yan, M. Kuang, Y.X. Xu, K. Wen, R. Lupoia, J. Mater. Sci. Technol. 35, 1003 (2019)

[36] P.V. Kiryukhantsev-Korneev, A.N. Sheveyko, N.V. Shvindina, E.A. Levashov, D.V. Shtansky, Ceram. Int. 44, 7637 (2018)

[37] X.Q. Liu, H. Cheng, Z.J. Li, H. Wang, F. Chang, W.G. Wang, Q.H. Tang, P.Q. Dai, Vacuum 165, 297 (2019)

[38] X.F. Li, Y.H. Feng, B. Liu, D.H. Yi, X.H. Yang, W.D. Zhang, G. Chen, Y. Liu, P.K. Bai, J. Alloys Compd. 788, 485 (2019)

[39] J. Liu, H. Liu, P.J. Chen, J.B. Hao, Surf. Coat. Technol. 361, 63 (2019)

[40] T. Chen, W.N. Wu, W.P. Li, D.F. Liu, Opt. Laser Technol. 116, 345 (2019)

[41] B.A. Khamidullin, I.V. Tsivilskiy, A.I. Gorunov, A.K. Gilmutdinov, Surf. Coat. Technol. 364, 430 (2019)

[42] A. Riquelme, P. Rodrigo, M.D. Escalera-Rodríguez, J. Rams, Opt. Laser. Eng. 78, 165 (2016)

[43] N. Tamanna, R. Crouch, S. Naher, Opt. Laser. Eng. 122, 151 (2019)

[44] M.N. Fesharaki, R.S. Razavi, H.A. Mansouri, H. Jamali, Surf. Coat. Technol. 353, 25 (2018) 
[45] S.T. Peng, T. Li, J.L. Zhao, S.P. Lv, G.Z. Tan, M.M. Dong, H.C. Zhang, J. Clean. Prod. 227, 58 (2019)

[46] L. Reddy, S.P. Preston, P.H. Shipway, C. Davis, T. Hussain, Surf. Coat. Technol. 349, 198 (2018)

[47] Z.I. Mahmoud Ibrahim, A.D.S. Ahmed, T.Y. Kuo, F. Yusof, M. Hamdi, Mater. Chem. Phys. 235, 1 (2019)

[48] C. Zhang, G.J. Chen, P.Q. Dai, Heat Treat. Met. 40, 146 (2015)

[49] M.H. Sha, L. Zhang, J.W. Zhang, N. Li, T.Z. Li, N. Wang, Rare Met. Mater. Eng. 46, 1237 (2017)

[50] R.D. Li, P.D. Niu, T.C. Yuan, P. Cao, C. Chen, K.C. Zhou, J. Alloys Compd. 746, 125 (2018)

[51] Z.C. Qiu, C.W. Yao, K. Feng, Z.G. Li, P.K. Chu, Int. J. Lightweight Mater. Manuf. 1, 33 (2018)

[52] M. Li, J. Gazquez, A. Borisevich, R. Mishra, K.M. Floresa, Intermetallics 95, 110 (2018)

[53] C. Zhang, B. Huang, P.Q. Dai, Chin. Surf. Eng. 29, 32 (2016)

[54] C. Zhang, B.Q. Wu, Q.T. Wang, D.N. Chen, P.Q. Dai, Rare Met. Mater. Eng. 46, 2639 (2017)

[55] C. Zhang, B. Huang, P.Q. Dai, Met. Heat Treat. 41, 82 (2016)

[56] H. Zhang, W.F. Wu, Y.Z. He, M.X. Li, S. Guo, Appl. Surf. Sci. 363, 543 (2016)

[57] G.J. Chen, C. Zhang, Q.H. Tang, P.Q. Dai, Rare Met. Mater. Eng. 44, 1418 (2015)

[58] Y.X. Guo, X.J. Shang, Q.B. Liu, Surf. Coat. Technol. 344, 353 (2018)

[59] Y. Shon, S.S. Joshi, S. Katakam, R.S. Rajamure, N.B. Dahotre, Mater. Lett. 142, 122 (2015)

[60] B. Zheng, Y. Jiang, W. Hu, Appl. Laser 36, 18 (2016)

[61] G.H. Meng, X. Lin, H. Xie, C. Wang, S.Q. Wang, X. Ding, J. Alloys Compd. 672, 660 (2016)

[62] S. Zhang, C.L. Wu, J.Z. Yi, C.H. Zhang, Surf. Coat. Technol. 262, $64(2015)$
[63] S. Zhang, C.L. Wu, J.Z. Yi, C. Wang, C.H. Zhang, M. Guan, J.Z. Tan, Chin. J. Lasers 41, 97 (2014)

[64] I. Kunce, M. Polanski, J. Bystrzycki, Int. J. Hydrogen Energy. 39, 9904 (2014)

[65] F. Zhou, Q.B. Liu, B. Zheng, Chin. J. Lasers 43, 58 (2016)

[66] B. Huang, C. Zhang, H. Cheng, Q.H. Tang, H.C. Rao, P.Q. Dai, Chin. Surf. Eng. 27, 82 (2014)

[67] Z.F. Huang, C. Zhang, Q.H. Tang, P.Q. Dai, B. Wu, Surf. Technol. 42, 9 (2013)

[68] Z. Chong, G. Chen, P. Dai, Heat Treat. Met. 40, 146 (2015)

[69] R. Ferro, A. Saccone, Physical Metallurgy (North Holland, Amsterdam, 1996)

[70] Z.J. Wang, Y.H. Huang, C.T. Liu, J.J. Li, J.C. Wang, Intermetallics 109, 139 (2019)

[71] M. Calvo-Dahlborg, S.G.R. Brown, J. Alloys Compd. 724, 353 (2017)

[72] S.Z. Li, H.R. Zhang, D.B. Dai, G.T. Ding, X. Wei, Y.K. Guo, J. Alloys Compd. 782, 110 (2019)

[73] M.G. Poletti, L. Battezzati, Acta Mater. 75, 297 (2014)

[74] X. Gu, Phys. Exp. Colloids 3, 66 (2006)

[75] J.W. Gooch, Relative Standard Deviation (Encyclopedic Dictionary of Polymers, West Yorkshire, 2011).

[76] H. Zho, L.R. Long, Adv. Psychol. Sci. 12, 942 (2004)

[77] T. Egami, Y. Waseda, J. Non-Cryst, Solids 64, 113 (1984)

[78] L. Vitos, I.A. Abrikosov, B. Johansson, Phys. Rev. Lett. 87, 1 (2001)

[79] T. Uesugi, K. Higashi, Comput. Mater. Sci. 67, 1 (2013)

[80] A. Takeuchi, A. Inoue, Mater. Sci. Eng. 304, 446 (2001)

[81] A. Takeuchi, A. Inoue, Mater. Trans. 41, 1372 (2000)

[82] M.M. Ren, B.S. Li, H.Z. Fu, Trans. Nonferrous Met. Soc. China 23, 991 (2013) 